\title{
Role of High-Resolution Computed Tomography in the Evaluation of Anatomical Variations of Sphenoid Sinus and Its Clinical Importance in FESS
}

\author{
Parvathy P. ${ }^{1}$
}

${ }^{1}$ Department of Radiodiagnosis, Sree Gokulam Medical College and Research Foundation, Venjaramoodu, Kerala, India.

\section{ABSTRACT}

\section{BACKGROUND}

Sphenoid sinus of all sinuses is generally the most inaccessible sinus to the surgeons. The trans-sphenoid route is considered to be the standard approach for surgery of pituitary adenomas. Knowing the details of the anatomy of sphenoid sinus and the extent of pneumatization can guide the surgeon through difficult corners of the approach. We wanted to evaluate the incidence of the different anatomical variations of sphenoid sinus as detected by HRCT scan and their impact on related neurovascular structures for the safe removal of inter sphenoid and pituitary lesions.

\section{METHODS}

This prospective study was conducted in Sree Gokulam Medical College \& Research Foundation, Trivandrum. Study population included 150 cases who were referred for HRCT of the paranasal sinuses to the Department of Radiodiagnosis, over a period of 12 months (from November 2018 to October 2019). After obtaining a written informed consent and history, all the patients underwent HRCT axial section of PNS. Once the axial sections were obtained through the paranasal sinuses, these images were reconstructed into coronal sections by multiplanar reconstruction (MPR) technique without exposing the patient.

\section{RESULTS}

Onodi cells were found predominantly in female patients with male:female ratio 2:7. Optic nerve protrusion and dehiscence had male predominance. Rest of the variations had no significant gender difference and few variations were seen equally distributed among both males and females. The most common variation observed in our study was pterygoid process pneumatization. Of the total 150 patients, pterygoid process pneumatization was identified in 76 patients including bilateral in 54 patients. Next common variation observed was vidian nerve protrusion, identified in 62 patients along with dehiscence of wall in 38 patients. Maxillary nerve protrusion was present in 42 patients and its dehiscence of wall in 10 patients. Optic nerve protrusion was identified in 41 patients and dehiscence of wall was noted in only 6 patients.

\section{CONCLUSIONS}

Sphenoid sinuses are the most inaccessible paranasal sinuses and are surrounded by significant anatomical structures such as the orbit and its contents, cavernous sinus, internal carotid artery (ICA) and the anterior cranial fossa. HRCT of sphenoid sinus for the demonstration of the anatomical variations and its relation to the vital adjacent crucial anatomical structures helps in reducing the complications during trans-sphenoidal surgeries and functional endoscopic sinus surgery. Our study of HRCT findings delineates most of the anatomical variations in sphenoid sinus and thus helps the surgeons in appropriate surgical planning and helps them to reduce the intraoperative complications.

\section{KEY WORDS}

High Resolution Computed Tomography, Sphenoid Sinus, Functional Endoscopic Sinus Surgery (FESS)

\author{
Corresponding Author: \\ Parvathy P., \\ Assistant Professor \\ Department of Radiodiagnosis, \\ Puthenpurackal, Noornad P. O., \\ Allapuzha District, 690504, \\ Kerala, India. \\ E-mail: drparvathyparu@gmail.com
}

DOI: 10.14260/jemds/2020/408

Financial or Other Competing Interests: None.

How to Cite This Article:

Parvathy P. Role of high-resolution computed tomography in the evaluation of anatomical variations of sphenoid sinus and its clinical importance in fess. J. Evolution Med. Dent. Sci. 2020;9(25): 1874-1880, DOI:

$10.14260 /$ jemds $/ 2020 / 408$

Submission 01-04-2020,

Peer Review 17-05-2020

Acceptance 23-05-2020,

Published 22-06-2020.

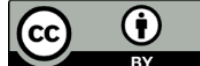




\section{BACKGROUND}

Sphenoid sinus is deeply seated in the skull and is the most inaccessible paranasal sinus. It is surrounded by vital structures such as the internal carotid artery, optic nerve and cavernous sinus. Development wise, sphenoid sinus emerges in the fourth month as evagination from posterior nasal capsule into the sphenoid bone. Sinus will obtain the adult configuration by the age of 10 to 12 years. The degree of pneumatization of sphenoid sinus varies \& depending on that the sinus is classified as non-pneumatized, pre-sellar and sellar. As a result of extensive pneumatization of sphenoid sinus vital structures like internal carotid artery, optic nerve, vidian nerve and maxillary nerve can be protruded into the sinus with or without bony walls. In our study we intend to evaluate the anatomical variations of sphenoid sinus and its effect on the adjacent neurovascular structure. Functional Endoscopic Sinus Surgery (FESS) needs detailed guiding anatomical map of the paranasal sinuses especially of the sphenoid sinus because of its critical location and large variations in its appearance.

\section{Objectives}

- To demonstrate the anatomical variations of sphenoid sinus and related structures.

- To establish the relations of the sphenoid sinus variations to adjacent crucial anatomical structures.

- To establish the impact of the HRCT findings of variations in sphenoid sinus during functional endoscopic sinus surgery for planning and to reduce the possible complications.

\section{METHODS}

This is a descriptive study conducted in Sree Gokulam Medical College during the period from November 2018 to October 2019. All the subjects during this period, who fulfilled the inclusion criteria were included in the study. Hospital based cross sectional study and descriptive statistics were applied to find out common anatomical variations in our study population of 150 patients.

After obtaining a written informed consent and history, all the patients underwent HRCT axial section of paranasal sinuses (PNS).Once the axial sections were obtained through the paranasal sinuses, these images were reconstructed into coronal sections by multiplanar reconstruction (MPR) technique without exposing the patient.

\section{Inclusion Criteria}

Patients above 15 years of age who were referred for HRCT of the paranasal sinuses to the Department of Radio diagnosis of Sree Gokulam Medical College are included in this study.

\section{Exclusion Criteria}

- $\quad$ Patients with prior surgery of paranasal sinuses (PNS).

- Patients with sinonasal tumours and head \& neck injuries.

In each case we analysed the following normal anatomical variants

- Pneumatization of pterygoid process, anterior clinoid process and greater wing of sphenoid.

- Protrusion of internal carotid artery, optic nerve, maxillary nerve and vidian nerve.

- Dehiscence of bony walls of internal carotid artery, optic nerve, maxillary nerve and vidian nerve.

- Patterns of septations, septal attachment to the bony bulges over the optic nerve and the internal carotid artery.

- $\quad$ Presence of Onodi cells (sphenoethmoid cells).

In our study,

- Presence of air density around the vidian nerve and maxillary nerve in at least one coronal section was considered as protrusion of vidian nerve and maxillary nerve.

- $\quad$ Presence of more than half of the circumference of ICA into the sinus cavity at any degree were considered as protrusion of ICA.

- Absence of visible bone density separating the sinus from the ICA, optic nerve, maxillary nerve and vidian nerve as dehiscence of bony wall.

- Pterygoid process pneumatization were recognized if it extends beyond a horizontal plane crossing the vidian canal and pneumatization of greater wing of sphenoid were considered if extension goes beyond vertical line crossing foramen rotundum / maxillary canal.

\section{RESULTS}

\section{Gender and Age Distribution}

During the period of one year of the study, 150 patients who fulfilled inclusion criteria were studied, out of which $72(48 \%)$ were female and $78(52 \%)$ were male. These patients were between the age group of 19-69 years of age.

\section{Pneumatization}

Anterior Clinoid Process- In our study, this variation was found in $36(24 \%) / 150$ patients and found bilaterally in $28(36 \%)$ patients. Right sided pneumatization of anterior clinoid process were noted in 5 patients and left sided in 3 patients. 19 male patients and 17 female patients had pneumatization of anterior clinoid process in our study.

Greater Wing of Sphenoid- Pneumatization of greater wing of sphenoid was found in $14(9.3 \%)$ patients out of all patients (150) in our study and was found bilaterally in 9/14 patients. Out of 14 cases, 8 patients were found among males and 6 among female. Right sided 
pneumatization of greater wing of sphenoid seen only in 2 $(14.3 \%)$ patients and left sided in $3(21.4 \%)$ patients.

Pterygoid Process- Pneumatization of pterygoid process was observed in $76(50.7 \%)$ patients out of total 150 patients and were found bilaterally in 54/76 patients. Out of total 76 patients, 37 patients were found among males and 39 among female (no significant change in distribution).Right sided pneumatization was seen in 13 (17.1\%)/76 patients and left sided pneumatization in 9 $(11.8 \%)$ patients.

\begin{tabular}{|c|c|c|c|c|c|c|c|c|}
\hline \multirow[b]{2}{*}{ Pneumatization } & \multicolumn{2}{|c|}{ Bilateral } & \multicolumn{2}{|c|}{ Right } & \multicolumn{2}{|c|}{ Left } & \multicolumn{2}{|c|}{ Total } \\
\hline & ठِ & $\begin{array}{l}\ddot{\Xi} \\
\stackrel{\Xi}{0} \\
\text { ڤ }\end{array}$ & $\stackrel{\Xi}{\Xi}$ & 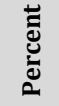 & $\stackrel{\Xi}{\Xi}$ & 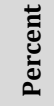 & $\stackrel{\Xi}{\Xi}$ & 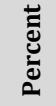 \\
\hline Anterior clinoid process & 28 & 77.8 & 5 & 13.9 & 3 & 8.3 & 36 & 100.0 \\
\hline Greater wing of sphenoid & 9 & 64.3 & 2 & 14.3 & 3 & 21.4 & 14 & 100.0 \\
\hline Pterygoid process & 54 & 71.1 & 13 & 17.1 & 9 & 11.8 & 76 & 100.0 \\
\hline
\end{tabular}

\section{Protrusion}

Internal Carotid Artery- A protruding internal carotid artery into the sphenoid sinus was found in 35 patients (23.3\%), of whom 15 (42.9\%) were bilateral, 12 (34.3\%) were right sided and 8 (22.9\%) were left sided. Out of 35 cases, 19 patients were males and 16 patients were females in our study.

Optic Nerve- The protrusion of the optic nerve was present in 41 patients $(27.3 \%)$. Protrusions were bilateral, right sided and left sided in 32 (78\%), $6(14.6 \%)$ and $3(7.3 \%)$ patients respectively. Optic nerve protrusion was observed in 24 male patients and 17 female patients.

Maxillary Nerve- The protrusion of the maxillary nerve (foramen rotundum) was encountered in 42 (28\%) patients, of whom 19 (45.2\%) were bilateral, 10 (23.8\%) were on the right side and 13 (31\%) were on the left side. Maxillary nerve protrusion were found equally distributed (21 patients each) among both male and female patients.

Vidian Nerve- A protruding vidian nerve into the sinus cavity was present in $62(41.3 \%)$ patients of whom 45 $(72.6 \%)$ were bilateral, $10(16.1 \%)$ were on the right side and $7(11.3 \%)$ were on the left side. Vidian nerve protrusion were observed equally distributed (31 patients each) among both male and female patients in our study.

\begin{tabular}{|c|c|c|c|c|c|c|c|c|}
\hline \multirow{2}{*}{ Protrusion } & \multicolumn{2}{|c|}{ Bilateral } & \multicolumn{2}{|c|}{ Right } & \multicolumn{2}{|c|}{ Left } & \multicolumn{2}{|c|}{ Total } \\
\hline & Count & $\%$ & Count & $\%$ & Count & $\%$ & Count & $\%$ \\
\hline $\begin{array}{c}\text { Internal carotid } \\
\text { artery }\end{array}$ & 15 & 42.9 & 12 & 34.3 & 8 & 22.9 & 35 & 100.0 \\
\hline Optic nerve & 3 & 78.0 & 6 & 14.6 & 3 & 7. & 41 & 100.0 \\
\hline Maxillary nerve & 19 & 45.2 & 10 & 23.8 & 13 & 31.0 & 42 & 100.0 \\
\hline Vidian nerve & 45 & 72.6 & 10 & 16.1 & 7 & 11.3 & 62 & 100.0 \\
\hline Con & isol & I & c & ع & $\sin$ & 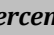 & & \\
\hline
\end{tabular}

\section{Dehiscence}

Internal Carotid Artery- Dehiscence of the bony wall of the internal carotid artery observed in 6 patients $(4 \%)$ of whom 3 (50\%) were bilateral, 1 (16.7\%) were on the right and $2(33.3 \%)$ were on the left. This variation seen in 2 males and 4 female patients in our study.

Optic Nerve- Dehiscence of the bony wall of the optic nerve (optic canal) was observed in $6(4 \%)$ patients, of whom 3 (50\%) were bilateral, 2 (33.3\%) were right sided and 1 $(16.7 \%)$ were left sided. Out of all patients with this variation, 5 patients were males and only 1 female patient, with male predominance in our study.

Maxillary Nerve- The protrusion of the maxillary nerve (foramen rotundum) was encountered in 42 (28\%) patients, of whom 19 (45.2\%) were bilateral, 10 (23.8\%) were on the right side and $13(31 \%)$ were on the left side. Maxillary nerve protrusion was found equally distributed (21 patients each) among both male and female patients.

Vidian Nerve- Dehiscence of the bony wall of the vidian nerve (canal) was identified in $38(25.3 \%)$ patients, of whom 50 (19\%), 11 (28.9\%) and 8 (21.1\%) were bilateral, right sided and left sided respectively. This variation found equally distributed (19 patients each) among both males and female patients in our study.

\begin{tabular}{|cccccccccc|}
\hline Dehiscence & \multicolumn{3}{c}{ Bilateral } & \multicolumn{3}{c|}{ Right } & \multicolumn{2}{c|}{ Left } & \multicolumn{2}{c|}{ Total } \\
Count & $\%$ & Count & $\%$ & Count & $\%$ & Count & $\%$ \\
Internal carotid & 3 & 50.0 & 1 & 16.7 & 2 & 33.3 & 6 & 100.0 \\
artery & & & & & & & & \\
Optic nerve & 3 & 50.0 & 2 & 33.3 & 1 & 16.7 & 6 & 100.0 \\
Maxillary nerve & 5 & 50.0 & 2 & 20.0 & 3 & 30.0 & 10 & 100.0 \\
Vidian nerve & 19 & 50.0 & 11 & 28.9 & 8 & 21.1 & 38 & 100.0 \\
\hline \multicolumn{1}{|c}{ Comparison of Dehiscence of Bony Wall of Artery } \\
and Nerves in Percentage \\
\hline
\end{tabular}

\section{Presence of Onodi Cells}

Presence of Onodi cells were found only in $9(6 \%)$ patients out of all 150 patients in our study. 7 cases of Onodi cells were seen in female patients and 2 in male patients, with female predominance in our study.

\section{Septations}

Presence of septations in sphenoid sinus were found in 133 patients, of whom 77 (57.4\%) patients had single septations and $56(42 \%)$ had multiple septations. 67 patients were males and 66 females with septations of sphenoid sinus in our study. In addition to the analysis of the number of septa, special emphasis is placed to their insertion in relation to the carotid and optic canal. Attachment of sphenoid septations to optic canal were seen in 32 (24\%) cases and to carotid canal in $12(9 \%)$ cases.

\begin{tabular}{|ccc|}
\hline Types of Septations & Single & Multiple \\
Positive Cases (out of 133 Cases) & $\mathbf{7 7}$ & $\mathbf{5 6}$ \\
& Optic Canal & Carotid Canal \\
Attachment & 32 & 12 \\
\hline Type of Sphenoid Septations and Attachment \\
to Optic Canal and Carotid Canal & \\
\hline
\end{tabular}



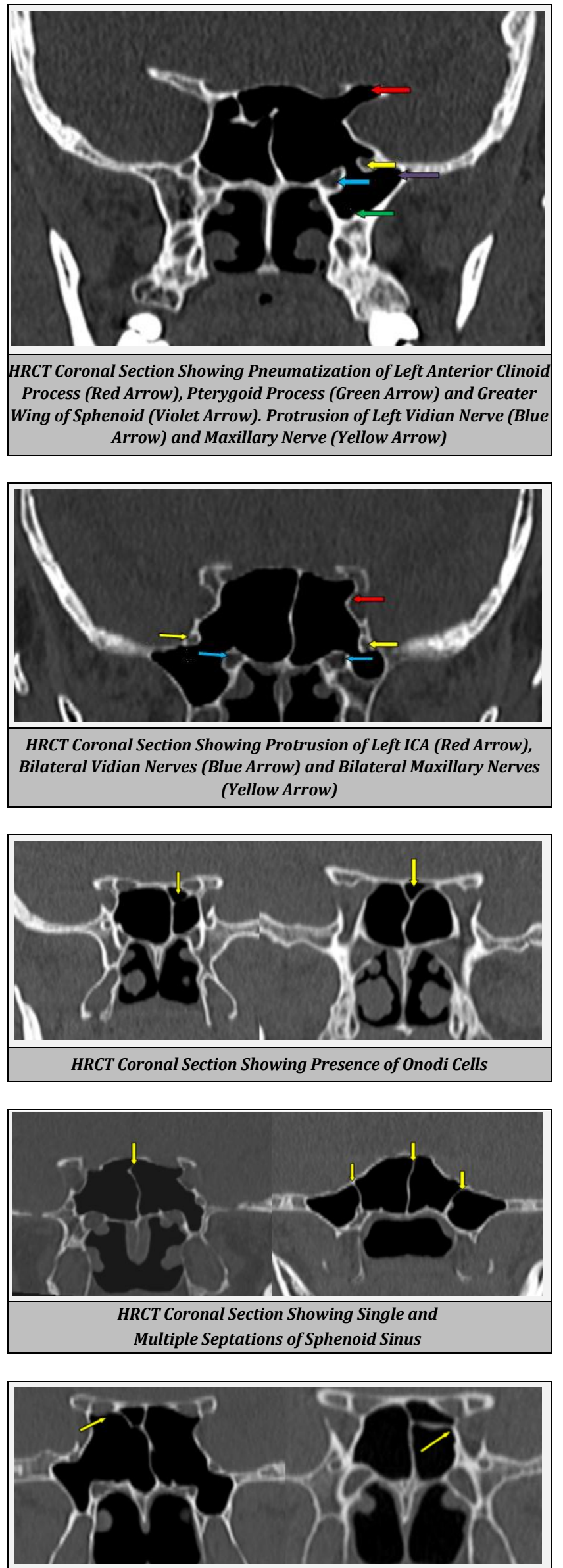

HRCT Coronal Section Showing Attachment of Septation to Right Optic Canal and Left Carotid Canal. There is Dehiscence of Bony Wall and Protrusion of Bilateral Optic Nerves. Also Noted on Right Side in Additional to Septal Attachment to Optic Canal

\section{DISCUSSION}

A total of 150 patients who met the study inclusion criteria were included in the analysis of data and compared variations in the anatomical configuration of surrounding structures including protrusion or dehiscence of internal carotid artery, optic nerve, vidian nerve and maxillary nerve; presence of pneumatization of pterygoid process, anterior clinoid process, greater wing of sphenoid; presence of Onodi cells; septations and its attachment to optic and carotid canal.

\section{Pterygoid Process}

Pterygoid process pneumatization is recognized if it extends beyond a horizontal plane crossing the vidian canal. In our study, pneumatization of the pterygoid process was found in 76 patients of whom 54, 13 and 9 were bilateral, right sided and left sided respectively. There is no significant change in distribution of this pneumatization among males and female patients (37 cases among males and 39 among female patients). Presence of Pterygoid process pneumatization is an important pathway for access to the central skull base. For instance, extended transnasal endoscopic approaches may reach the pterygoid process through the medial part of the posterior maxillary wall. These techniques may provide a route for endoscopic repair of cerebrospinal fluid leaks and endoscopic biopsy of skull base lesions. Such information may be important in preoperative planning for skull base surgery. Pneumatization of pterygoid process thins the bony floor of the scaphoid fossa to as little as $0.2 \mathrm{~mm}$ producing an intimate relation between the sinus and the auditory tube.

\section{Anterior Clinoid Process}

In our study reports pneumatization of anterior clinoid process was encountered in 36 patients. It was found bilateral in 28 patients, on the right side in 5 patients and on left side in 3 patients. 19 male patients and 17 female patients had pneumatization of anterior clinoid process in our study. The prevalence of anterior clinoid process pneumatization has been well documented in the literature. Bolger et al. ${ }^{1}$ found anterior clinoid process pneumatization in $13 \%$ of 202 paranasal sinus CT scans. In a review of 150 paranasal sinus CT scans, De Lano et $\mathrm{al}^{2}{ }^{2}$ found anterior clinoid process pneumatization in only 13 of 300 sides (4\%). Pneumatization of anterior clinoid process forms the opticocarotid recess, i.e. the small space on the lateral wall of the sphenoid sinus, between the optic canal superiorly and the carotid prominence inferiorly. The optico-carotid recess is supposed to concur with ipsilateral optic nerve and/or internal carotid artery protrusion into the sphenoid sinus. We observed pneumatization of anterior clinoid process in our study series as well.

\section{Greater Wing of Sphenoid}

We observed pneumatization of greater wing of sphenoid in 14 patients, of whom 9 were bilateral, 2 were on the right side and 3 were on the left side. The distribution of this pneumatization was observed in 8 male patients and 6 
female patients. We defined pneumatization of greater wing of sphenoid as extension beyond a vertical line crossing foramen rotundum. Pneumatization of the floor of the middle cranial fossa in the presence of arachnoid granulations along the inner surface of the greater wing of the sphenoid, where this appendage forms the anterior wall of the middle cranial fossa. John Earwaker ${ }^{3}$ discovered pneumatization of greater wing of sphenoid in $10.7 \%$ of patients. These granulations form "pit-holes" on the floor of the middle cranial fossa and although they are not pathologic in and of themselves, enlargement of these pits has been casually implicated in the development of non-traumatic cerebrospinal leaks.

\section{Internal Carotid Artery}

We found protrusion of internal carotid artery into the sphenoid sinus in 35 patients, of whom 15 were bilateral, 12 were right sided and 8 were left sided. Out of 35 patients with ICA protrusion, 19 patients were males and 16 patients were females in our study. Dehiscence of the bony wall of internal carotid artery was seen in 6 patients of whom 3 were bilateral, 1 were on the right and 2 were on the left. This variation were observed in 2 males and 4 female patients in our study. Fuji et al. ${ }^{4}$ studied 25 cadaver sphenoid bones and found $8 \%$ of carotid arteries dehiscent of bone in the lateral sphenoid. Kennedy et al. ${ }^{5}$ found dehiscence on the bony wall of the internal carotid artery in $25 \%$ of patients. Occasionally they found the artery only with a mucoperiosteal covering coursing through the sphenoid sinus. In our present study, presence of the circumference into the sinus cavity at any degree was enough to define protrusion. If the surgeon is unaware of dehiscence or protrusion of the artery, even fatal haemorrhage may happen because it is difficult to control bleeding from an injured internal carotid artery within the sphenoid sinus. Sphenoid sinus infection may also make dehiscent or protruded internal carotid artery vulnerable to damage. ${ }^{6}$

\section{Optic Nerve}

In our study, the protrusion of the optic nerve was found in 41 patients. Protrusions were bilateral, right sided and left sided in 32, 6 and 3 patients respectively. Optic nerve protrusion was observed in 24 male patients and 17 female patients. Dehiscence of the bony wall of the optic canal was observed in 6 patients of whom 3 were bilateral, 2 were right sided and 1 were left sided. Out of 6 patients with dehiscence of bony wall of optic nerve, 5 patients were males and only 1 female patient, with male predominance in our study. However, previous studies reported a wide range of protrusion rates of 8 to $70 \% .^{7}$ Fuji et al. ${ }^{4}$ found that $4 \%$ of optic nerves were dehiscent of bone in the lateral sphenoid. They also reported that most optic nerves were covered by thin bone, measuring $0.5 \mathrm{~mm}$ or less in $78 \%$ of cases. They attributed the difference in the prevalence of anatomic variations to ethnic background. In the case of protrusion or dehiscence, optic nerve injury can occur due either to surgical trauma or as a complication of sinus disease. The risk of blindness is high if the surgeon damages the nerve within the sinus. ${ }^{8}$

\section{Maxillary Nerve}

We observed maxillary nerve protrusion in 42 patients, of whom 19 were bilateral, 10 were on the right side and 13 were on the left side. Maxillary nerve protrusion were found equally distributed ( 21 patients each) among both male and female patients. Dehiscence of the bony wall of the maxillary canal was seen in 10 patients, of whom 5 were bilateral, 2 were right sided and 3 were left sided. Dehiscence of bony wall of maxillary nerve were observed equally distributed ( 5 patients each) among both males and female patients in our study. In endoscopic sphenoid surgery, a protruded or dehiscent maxillary nerve is liable to iatrogenic injury. Furthermore, neuritis of a dehiscent maxillary nerve may result from sphenoid sinusitis and present as trigeminal neuralgia. ${ }^{9}$

\section{Vidian Nerve}

In our study, protruding vidian nerve into the sinus cavity was present in 62 patients of whom 45 were bilateral, 10 were on the right side and 7 were on the left side. Vidian nerve protrusion were observed equally distributed (31 patients each) among both male and female patients in our study. Dehiscence of the bony wall of the vidian canal was identified in 38 patients, of whom 19, 11 and 8 were bilateral, right sided and left sided respectively. These variations were found equally distributed (19 patients each) among both males and female patients in our study. Lang and Keller ${ }^{10}$ reported that the vidian nerve was protruded into the sinus cavity in $18 \%$. Pandolfo et al. ${ }^{11}$ emphasized that there is a variable relationship between the vidian canal and the sphenoid sinus. They also suggested that the vidian nerve can cause a clinical syndrome characterized by pain referred deeply in the nasal cavity (vidian neuralgia). Anatomic relationship of the vidian canal to the sphenoid sinus cavity help in decreasing the complication of the endoscopic transsphenoidal and vidian neurectomy surgery.

\section{Onodi Cells}

In our study, presence of Onodi cells was seen in 9 cases out of 150 patients. 7 cases of Onodi cells were seen in female patients and 2 in male patients, with female predominance in this study. Onodi cells are posterior ethmoidal cells that lies superior to the sphenoid sinus and is in close proximity to at least one optic nerve or internal carotid artery (ICA). Identification of the Onodi cell is therefore imperative to minimize perioperative morbidity due to its close proximity to the optic nerve and ICA.

\section{Septations and its Attachment}

The septations of the sphenoid sinus were found to be variable. Usually one intersphenoid septum separates sphenoid sinus in to two parts, but multiple septations also not uncommon. In our study, presence of septations were present in 133 patients of whom 77 patients had single septations and 56 had multiple septations. Out of 133 cases, 67 patients were males and 66 female patients with septations of sphenoid sinus in our study. Attachment of septation to carotid canal found in 12 patients and to optic canal in 32 patients. Karpur et al ${ }^{12}$ found septation of 
sphenoid sinus and its clinical significance in a retrospective study of the CT analysis of the paranasal sinuses in 200 patients. They reported multiple septations in $32 \%$ of male patients and $22.1 \%$ of female patients. Attachment of septation to the carotid canal seen in $19.4 \%$ of male and $16.8 \%$ female patients. In this situation, use extreme caution while removing the septum in order to prevent accidental and disastrous injury to the carotid artery. The sphenoid septum is an important landmark during the endonasal endoscopic transsphenoidal approach to important structures such as the carotid artery, optic canal and skull base.

\section{CONCLUSIONS}

Sphenoid sinuses are the most inaccessible paranasal sinuses and are surrounded by significant anatomical structures such as the orbit and its contents, cavernous sinus and ICA and the anterior cranial fossa. Only thin plates of bones separate these structures from the sphenoid sinus. Pneumatization of sphenoid sinuses ranged from their absence to extensive. According to the extent of sinus pneumatization, the bone covering the carotid arteries and optic nerves can be thin or even absent, making these structures susceptible to iatrogenic injury.

Computed tomography of the sphenoid sinus has improved the visualisation of sphenoid sinus anatomy and has allowed greater accuracy in evaluating sphenoid sinus and adjacent neurovascular structures. Preoperative CT examination of the sphenoid sinus can help to plan the safest and most direct route to the sella and parasellar region and can detect anatomical variations that increase the risk for intraoperative complications, including vision loss, haemorrhage and cerebrospinal fluid (CSF) leak. Endoscopic endonasal transsphenoidal approach (EEA) to treat sellar, parasellar and suprasellar tumours continues to gain significance. Due to the close proximity of the sphenoid sinus to the carotid artery and the optic canal, it is very important for surgeons to know the anatomical features and variations of the sphenoid sinus as relevant to EEA.

If the surgeon is unaware of dehiscence or protrusion of the internal carotid artery, fatal haemorrhage may happen. Sphenoid sinus infection may also make a dehiscent or protruded internal carotid artery vulnerable to damage. In case of protrusion or dehiscence, optic nerve injury can occur due to either surgical trauma or as a complication of sinusitis leading to high risk of blindness. Risk of blindness is high if the surgeon damages the nerve within the sinus. ${ }^{8}$ Compression of optic nerve can cause ischemia and venous congestion of the nerve. Furthermore, optic canal is the place where optic nerve is least nourished, which makes it very susceptible to injury. ${ }^{13}$ In endoscopic sphenoid surgery, a protruded or dehiscent maxillary nerve is liable to iatrogenic injury. Furthermore, neuritis of a dehiscent maxillary nerve may result from sphenoid sinusitis and present as trigeminal neuralgia. ${ }^{10}$ Anatomical relationship of the vidian canal to the sphenoid sinus cavity helps in decreasing the complication of the endoscopic transsphenoidal and vidian neurectomy surgery. Vidian nerve can cause a clinical syndrome characterized by pain referred deeply in the nasal cavity (vidian neuralgia).

Identification of the Onodi cell is necessary to minimize perioperative morbidity due to its close proximity to the optic nerve and ICA. Injury to internal carotid artery or optic nerve is a serious complication of transsphenoidal surgery. Comprehensive knowledge of the variable regional anatomy of the sphenoid sinus will undoubtedly reduce the surgical complications associated with transsphenoidal and functional endoscopic sinus surgery. Presence of anatomical variation does not mean a predisposition to sinus pathology, but these variations may predispose patients to increased risk of intraoperative complications. Radiologist must pay close attention to anatomical variations in the preoperative evaluation and provides a road map to the surgeon and help avoid possible complications and improve success of management strategies.

\section{REFERENCES}

[1] Bolger WE, Butzin CA, Parsons DS. Paranasal sinus bony anatomic variations and mucosal abnormalities: CT analysis for endoscopic sinus surgery. Laryngoscope 1991;101(1 Pt 1):56-64.

[2] DeLano MC, Fun FY, Zinreich SJ. Relationship of the optic nerve to the posterior paranasal sinuses: a CT anatomic study. Am J Neuroradiol 1996;17(4):669-75.

[3] Earwaker J. Anatomic variants in sinonasal CT. Radiographics 1993;13(2):381-415.

[4] Fuji K, Chambers SM, Rhoton AL. Neurovascular relationships of the sphenoid sinus. A microsurgical study. J Neurosurg 1979;50(1):31-9.

[5] Kennedy D, Zinrich H, Hassab M. The internal carotid artery as it relates to endoscopic shenoethmoidectomy. Am J Rhinol 1990;4:712.

[6] Sirikci A, Bayazit YA, Bayram M, et al. Variations of sphenoid and related structures. Eur Radiol 2000;10(5):844-8.

[7] Rosen MR, Saigal K, Evans J, et al. A review of the endoscopic approach to the pituitary through the sphenoid sinus. Curr Opin Otolaryngol Head Neck Surg 2006;14(1):6-13.

[8] Maniglia AJ. Fatal and major complications secondary to nasal and sinus surgery. Laryngoscope 1989;99(3):27683.

[9] Chong VF, Fan YF, Lau DP. Imaging the sphenoid sinus. Austrlas Radiol 1994;29:47-54.

[10] Lang J, Keller $H$. The posterior opening of the pterygopalatine fossa and the position of the pterygopalatine ganglion. Gegenbaurs Morphol Jahrb 1978;124(2):207-14.

[11] Pandolfo I, Gaeta M, Blandino I, et al. The radiology of pterygoid canal: normal and pathologic findings. AJNR Am J Neuroradiol 1987;8(3):497-83. 
[12] Kapur E, Kapidžić A, Kulenović A, et al. Septation of the sphenoid sinus and its clinical significance. International Journal of Collaborative Research on Internal Medicine \& Public Health 2012;6(10):1793-802.
[13] Sofferman RA, Harris P. Harris P. Mosher award thesis. The recovery potential of the optic nerve. Laryngoscope 1995;105(7 Pt Suppl 72):1-38. 